\title{
LONGITUDINAL WAVES THROUGH THE EARTH'S CORE*
}

\author{
By M. E. Denson, JR.
}

\begin{abstract}
Amplitudes, periods, and travel times of the longitudinal $\mathrm{P}^{\prime}$ or PKP core waves have been investigated. Results indicate that the epicentral distance of the main focal point and the travel time of $\mathrm{P}^{\prime}$ phases vary with the periods of the waves. This variation would seem reasonably explained in terms of dispersion. The point of reversal in the travel-time curve of the waves through the outer core is believed to lie near $157^{\circ}$. Data suggest a discontinuity between $120^{\circ}$ and $125^{\circ}$ rather than $110^{\circ}$. Anomalies existing in energy, period, and traveltime relationships of the $\mathrm{P}^{\prime}$ phases indicate that current concepts of velocity distribution and of propagation paths within the core are in need of modification.
\end{abstract}

\section{INTRODUCTION}

Since presentation of Oldham's controversial evidence in 1906, seismologists have come to accept the existence of an earth core and to recognize its effect on the paths of earthquake waves. A generalized form of the travel-time curve for the core phases called $\mathrm{P}^{\prime}$, and their corresponding simplified path relationships, are shown in figure 1 . The present study applies to the $\mathrm{P}^{\prime}$ phases.

The hypothesis of a high-velocity "inner" core is attributed to Lehmann (1937).$^{1}$ Calculations by Gutenberg and Richter $(1938,1939)$ and by Jeffreys (1939 a,b) have corroborated Miss Lehmann's hypothesis. Those phases called $\mathrm{P}^{\prime \prime}$ which are recorded at shorter distances are thus recognized as being reflected or refracted from the "inner" core or its transitional border zone. However, calculations of the velocity distribution at the "inner" core transition zone by Jeffreys and by Gutenberg and Richter are not in accord. Gutenberg and Richter have calculated an increase in velocity at the "inner" core contact where Jeffreys has calculated a decrease in velocity. Results of these calculations are reproduced in figure 2 . This particular velocity distribution determines the existence of the reversed segment CD in figure 1, $a$, and also, whether the $\mathrm{P}^{\prime \prime}$ phases at shorter distances are reflected or refracted. Jeffreys presents evidence in favor of reflected phases, whereas Gutenberg and Richter favor refracted phases.

This investigation has been directed to clarify, so far as is possible, the discrepancies noted above and to evaluate energy variations in terms of possible path relationships.

To this end, observations of amplitude, period, and arrival time of the $\mathrm{P}^{\prime}$ and associated phases have been recorded. Materials used in this study include seismograms from Pasadena and its network of stations and from Tucson and Huancayo. In addition, readings of amplitude, period, and time were taken from La Paz and Jena bulletins. Data from short-period and long-period recordings were analyzed separately. Phase travel times were computed on the basis of origin times given by Gutenberg and Richter (1949).

The method whereby observed and theoretical displacement ratios were

* Manuscript received for publication June 23, 1950.

${ }^{1}$ See "References" at the end of this paper. 


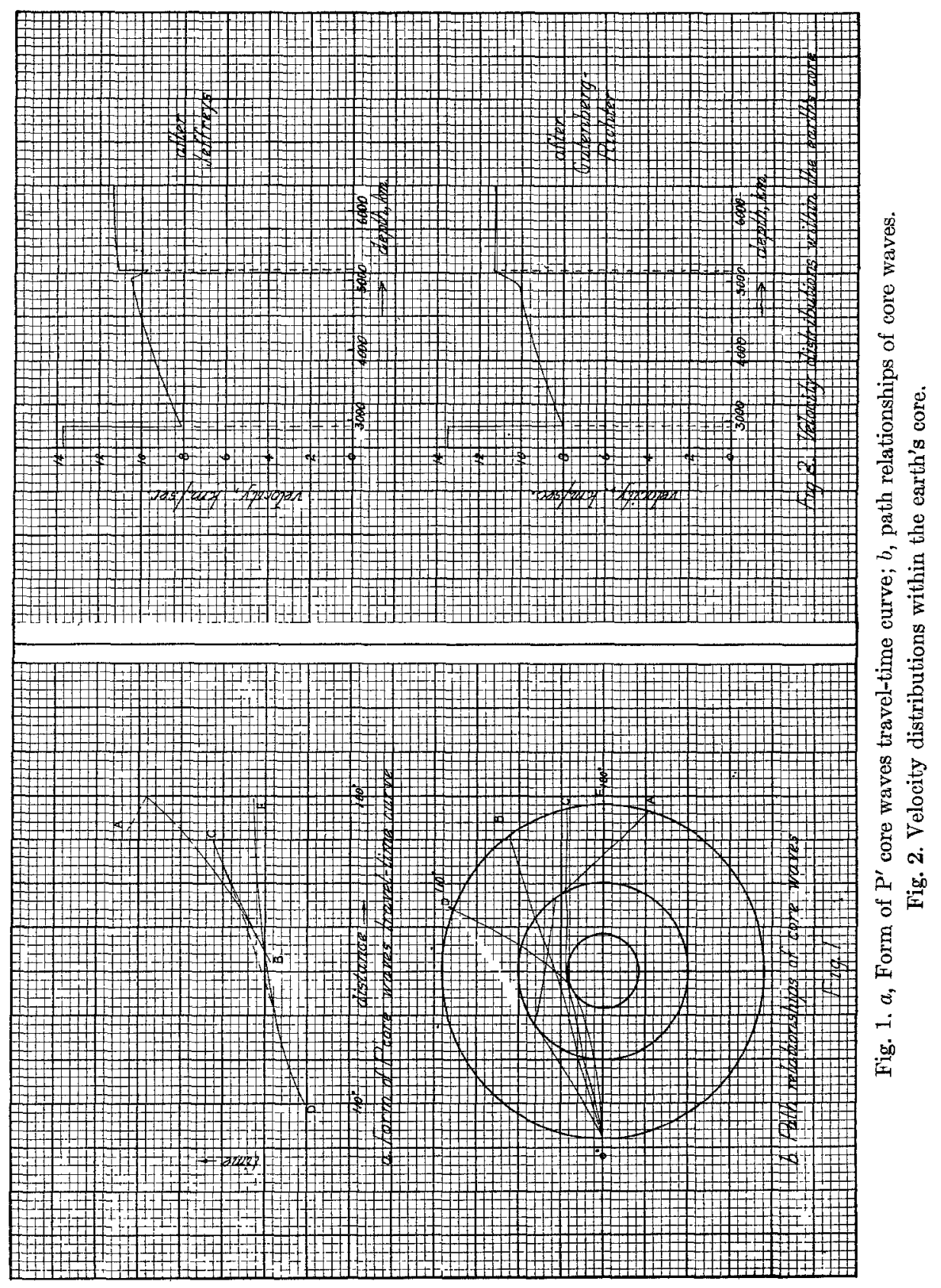


compared is that given by Gutenberg $(1944$, p. 85$)$. The basic formula for this comparison is given as

$$
\frac{u, w}{T} \times \frac{1}{k \sqrt{E}}=Q_{H, z} \sqrt{\frac{\left(F_{1} F_{2}\right) a \sin i_{k} d i_{h} / d \Delta}{\sin \Delta \cos i_{0}}}
$$

The derivation of this equation based upon the original theory of Zoeppritz may be found in the original paper of Zoeppritz, Geiger, and Gutenberg (1912). The quantity under the radical on the right-hand side is directly proportional to the energy which arrives at the earth's surface per unit area at arc distance $\Delta$, from a wave front with surface angle of incidence $i_{0}$. In the quantity $k \sqrt{E}$, $k$ depends on the fraction of total energy $E$ which goes into compressional or distortional motion at the source. The factors $F_{1}, F_{2}$ are proportional to the amount of incident energy refracted or reflected at a discontinuity. Evaluation of the $F$ factors for core refractions has been carried out by Dana (1944) following the energy relations established by Knott (1899). The quantity $Q_{H, Z}$ relates the distribution to vertical $(Z)$ and horizontal $(\mathrm{H})$ seismographs of incident energy arriving under different angles of incidence. Evaluation of this factor has been carried out by Gutenberg (1944). Effects of absorption are contained in the factor " $a$ ". Gutenberg (1945) has determined the absorption coefficient for core waves to have a value of $0.00012 / \mathrm{km}$. By assuming for shallow-depth earthquakes that $i_{h}$, the angle at which a ray leaves the source, is equal to $i_{0}$, the surface angle of incidence of that ray, a theoretical parameter $A_{T}$ may be defined. A corresponding observed parameter $A_{0}$ may be compared with the theoretical parameter $A_{T}$. Equations which define these parameters are:

$$
\begin{aligned}
& A_{T}=C^{\prime}-\log k \sqrt{E}-\log \frac{u, w}{T}=C-\log Q_{B, z} \sqrt{\frac{\left(F_{1} F_{2} a \tan i_{0} d i_{0} / d \Delta\right.}{\sin \Delta}} \\
& A_{0}=M-\log \frac{u, w}{T}-G(M-7)
\end{aligned}
$$

Definition of $A_{T}$ by equation (1) assumes plane motion in media which are isotropic between discontinuities. We infer from isotropy that motion may be purely compressional and purely shear. A further assumption is that from a point source energy is propagated equally in all directions. These assumptions, known to be invalid, have been incorporated in nearly all treatments of this subject. Their use can be reconciled only upon their sufficiency in providing a first approximation. Variations which may be found to exist between theory and observation must be partly considered in this light. The quantity $C$ has been determined statistically by Gutenberg (1945) to have a value of 6.3 . Magnitude $M$ was defined empirically by Richter (1935). The quantity $G(M-7)$ assumes variation in the duration of a phase with variation in magnitude. $G$ equals 0.1 for earthquakes of 6.5 to 7.5 magnitude, while 0.25 is used for earthquakes of magnitude greater than 7.5. Theoretical and ob- 


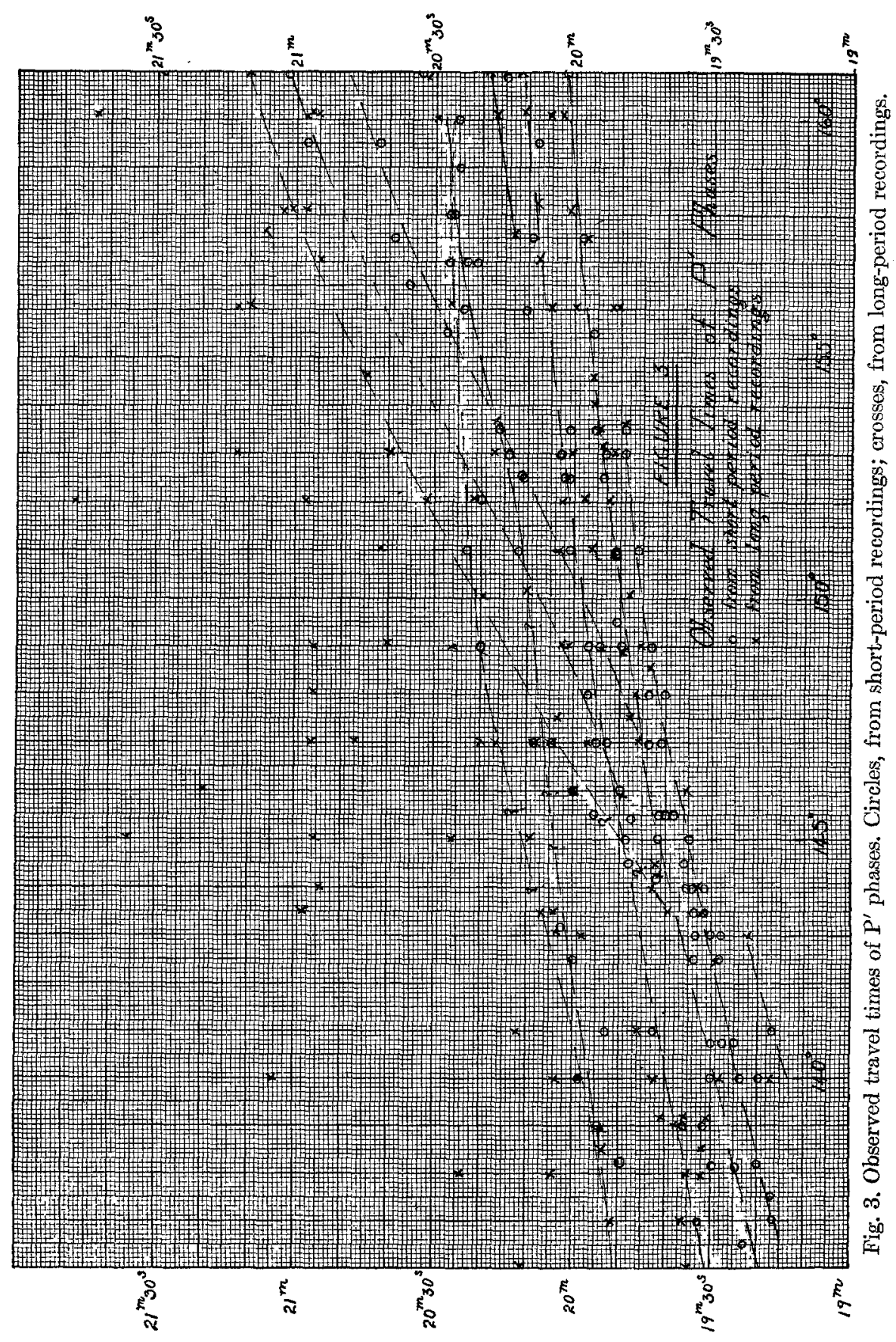


served displacement to period ratios, $u, w / T$ ( $u$ horizontal, $w$ vertical), as defined by equations (1) and (2), are in comparable form.

\section{General Relationships}

To resolve apparent complexities which arose in the process of recording $\mathrm{P}^{\prime}$ data, phase travel times were determined and plotted. Results of this analysis, partly shown in figure 3 , have disclosed segments of three groups of waves.

The first and earliest-arriving group is recorded from $110^{\circ}$ to $176^{\circ}$ with large amplitude/period ratios by short-period instruments. From $145^{\circ}$ to about $157^{\circ}$ the $\mathrm{P}_{1}^{\prime}$ phase of this group is also prominently recorded by long-period instruments. From energy considerations the focal point of this group for short-period instruments is believed to lie near $147^{\circ}$. No prominent focal point is recorded in this group by long-period instruments. The $\mathrm{P}^{\prime \prime}$ arrival times for this earliest-arriving group do not appear to lie on a continuous line. Traveltime curves for $\mathrm{P}^{\prime \prime}$ phases appear to denote a series of overlapping, en échelon segments. The $147^{\circ}$ focal point does not appear to occur earlier in time than the associated $\mathrm{P}^{\prime \prime}$ segment.

The second-arriving group of phases is well observed between $130^{\circ}$ and about $160^{\circ}$ for $\mathrm{P}^{\prime \prime}$ and $\mathrm{P}_{1}^{\prime}$ phases. At distances shorter than $130^{\circ}$ scattered observations may belong to motion of this group. On the whole, $\mathrm{P}_{2}^{\prime}$ phases for this group are few, but appear more prominent on long-period recordings. For this group large energies are recorded near $145^{\circ}$ by long-period instruments. Short-period instruments do not indicate energies which are abnormally large at $145^{\circ}$.

The third and latest-arriving group is also well observed between $130^{\circ}$ and about $160^{\circ}$ for $\mathrm{P}^{\prime \prime}$ and $\mathrm{P}_{1}^{\prime}$ phases. The $\mathrm{P}_{2}^{\prime}$ phases of this group are generally recorded strongly only by long-period instruments and with rather widely scattered arrival times. $\mathrm{P}^{\prime}$ and $\mathrm{P}^{\prime \prime}$ phases are here generally recorded prominently by long-period instruments. The position of the focal point of this group is not definitely known. In the neighborhood of the focal point only long-period recordings indicate large energy receptions. The shortest distance at which large amplitudes were noted for but few observations was about $143^{\circ}$; the longest distance was $147^{\circ}$. These observations varied in time of arrival by a span of about 15 seconds.

Scattered examples of the different phase motions are shown in figures 4,5 , and 6 .

The variation in energy among $\mathrm{P}^{\prime}$ groups with period and time suggests that dispersion may here be a dominant factor. The observed and unsmoothed travel times have been further analyzed by the method of Wadati and Masuda (1934) to evaluate the dispersion noted. It can only be stated that the long-period motion and the short-period motion of the $\mathbf{P}^{\prime}$ groups which are recorded at a given distance appear to enter the core at different angles of incidence and at different locations. Insufficient data and analyses as yet preclude definite determination of the type of dispersion which might produce the observed variations. Presumably, dispersion might arise under several sets of conditions, which may include absorption, velocity distributions at 


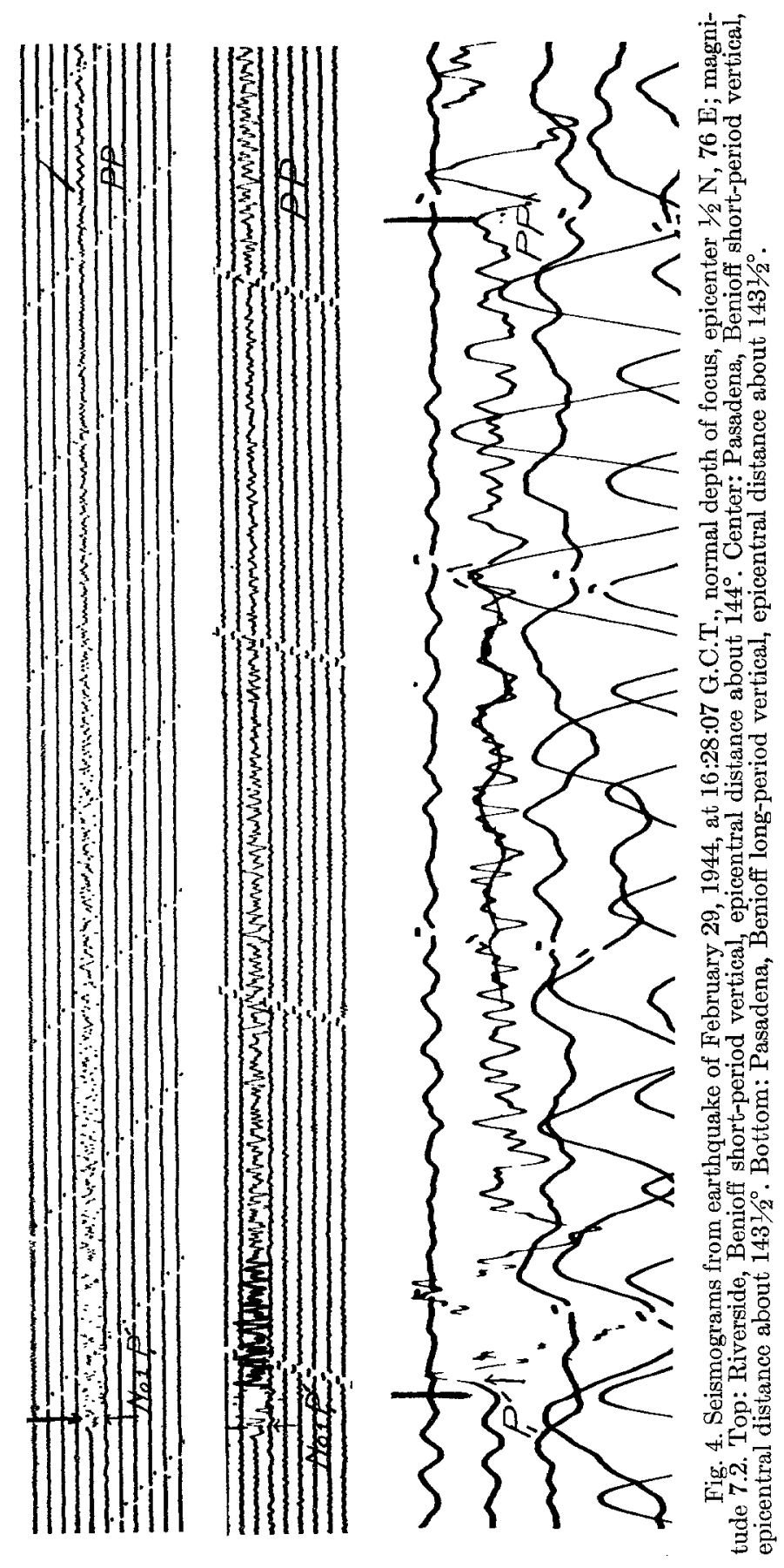




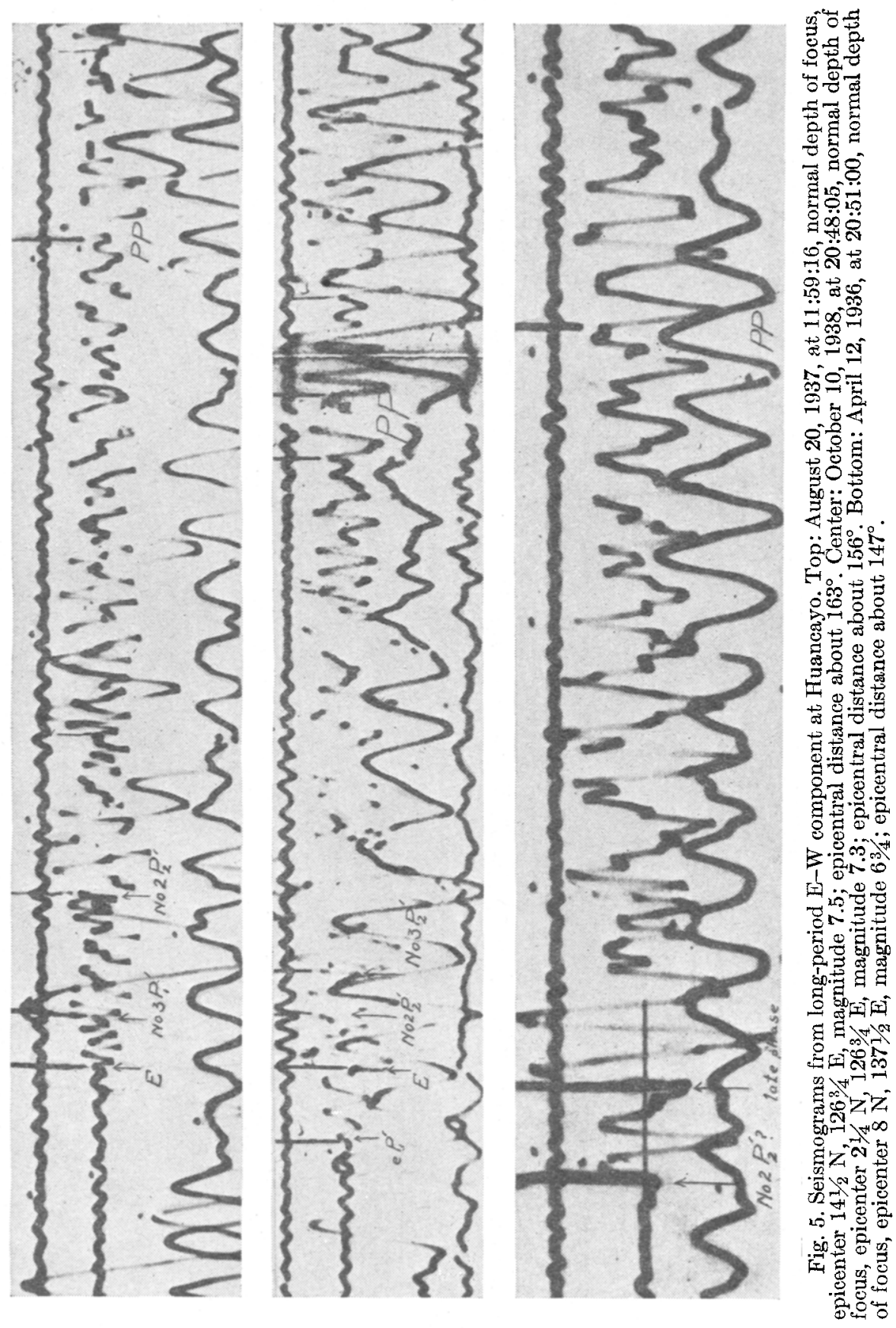




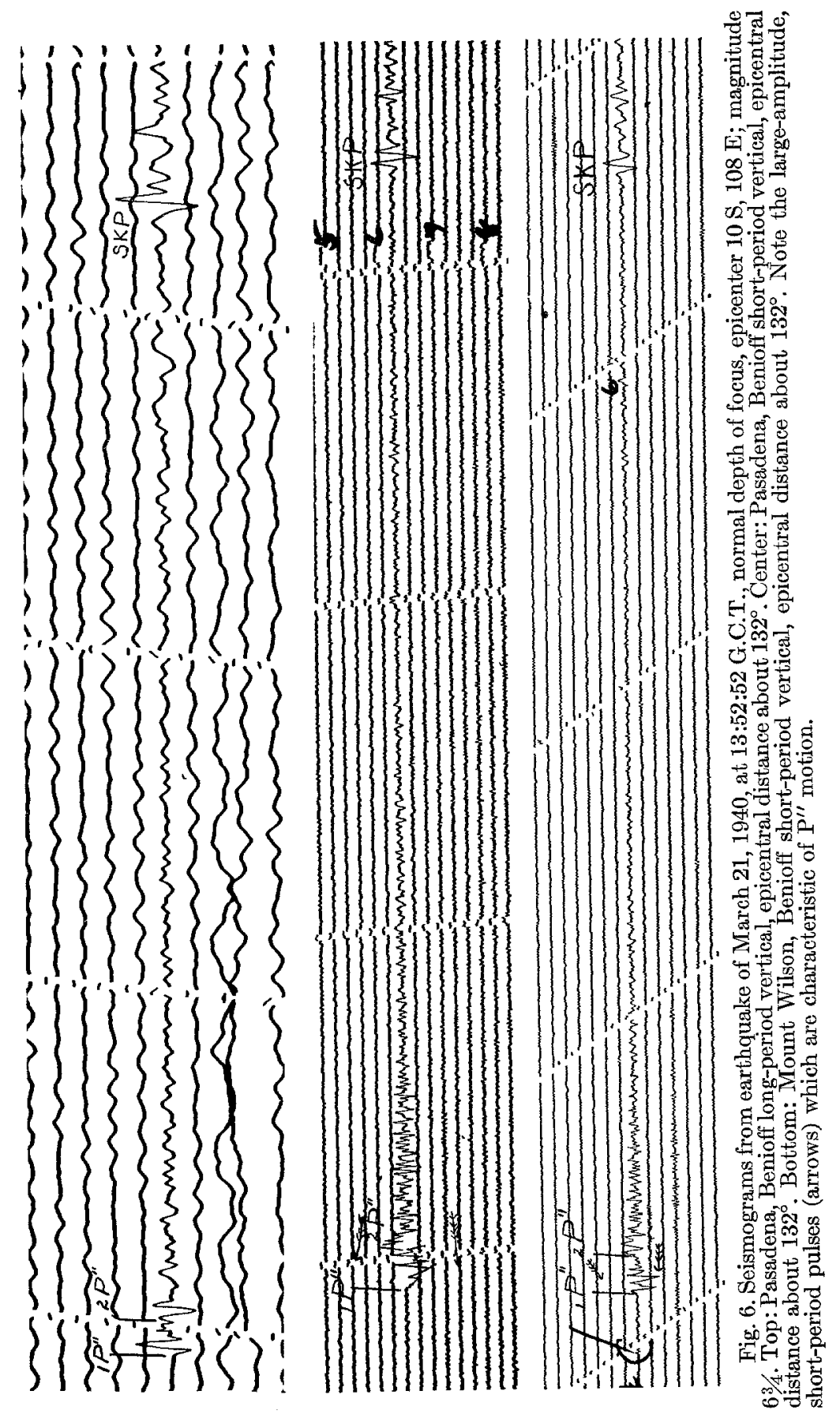


the contact zone of the core and of the "inner" core, or variations in the reaction of the material in the core to motions of different frequency. Spreading of the initial impulse into wave groups of maximum disturbance with differing component frequencies which propagate with differing group velocities might result in the variations observed.

Computed values of theoretical displacement ratios of $\mathrm{P}^{\prime \prime}$ and $\mathrm{P}_{1}^{\prime}$ to $\mathrm{PP}$, $\frac{(u, w / T) P^{\prime \prime}, P_{1}^{\prime}}{(u, w / T) P P}$, are plotted on logarithmic paper in figure 7 . Observed values of the same ratios are shown in figures 8 and 9 . By virtue of similarity between theoretical and observed values which were determined for the phase PP, discrepancies between theory (fig. 7) and observation (figs. 8 and 9) may be attributed largely to $\mathrm{P}^{\prime \prime}$ and $\mathrm{P}_{1}^{\prime}$. In addition to indications from individual phase data for $A_{0}$, equation (2), as shown in figure 10, displacement-ratio data indicate that the larger part of the energy in all the $\mathrm{P}^{\prime}$ phases is contained in the short-period components.

Other pertinent features shown by these data are:

1. Anomalous energies in $\mathrm{P}^{\prime \prime}$, depending upon the epicentral distance and the recorded period of the motion and rapid rise in $\mathrm{P}^{\prime \prime}$ energy around $120^{\circ}$.

2. Variation in position of the main focal point, depending on the recorded period of the waves.

3. A shadow zone at angular distances greater than $157^{\circ}$, a diminution in the amount of short-period energy in waves recorded in the distance interval between $147^{\circ}$ and $157^{\circ}$, and the suggested end of a long-period phase or abrupt drop in long-period energy near $157^{\circ}$.

Periods of the main energy-carrying phases of the $\mathrm{P}^{\prime \prime}$ and $\mathrm{P}_{1}^{\prime}$ waves have been plotted versus distance. These data plotted in figure 11 show a discontinuous increase in average period with distance. The over-all increase may be reasonably understood in the light of path relationships as shown by Gutenberg and Richter (1939, p. 123). Yet, the period data suggest a discontinuity between $120^{\circ}$ and $125^{\circ}$. This suggestion is strengthened by corresponding anomalies in travel-time data, and in observed data- $A_{0}$, equation (2) of figure 10,- and by the ratio data shown in figures 8 and 9 . Analyses thus far made suggest that a shadow zone occurs at distances shorter than about $120^{\circ}$ and that phase reinforcement or intersection occurs near $120^{\circ}$ and possibly near $132^{\circ}$ also. The observations are subject to further uncalculated complexities arising from the fact that at shorter distances the $\mathrm{P}^{\prime \prime}$ waves may possibly suffer appreciable loss of energy from transverse-wave generation at the inner-core contact. To evaluate the discontinuity near $120^{\circ}$, the depth of penetration of the $\mathrm{P}^{\prime \prime}$ ray arriving there was calculated. From the travel-time slope of $2.3 \mathrm{sec} /$ degree as determined from observations and the velocity distribution given by Bullen $\left(1947\right.$, p. 211 ) the $120^{\circ}$ ray appears to penetrate to a depth of about $4,990 \mathrm{~km}$. This is about the depth at which Jeffreys has calculated a velocity decrease (followed by a velocity increase), and at which Gutenberg and Richter have calculated a velocity increase. To arrive at distances shorter than $120^{\circ}$ would necessitate that the $\mathrm{P}^{\prime \prime}$ waves be reflected from the "inner" 
core. This could be realized by reason of a velocity decrease over a finite depth interval followed by an abrupt increase. In this case, refracted phases extend from about $120^{\circ}$ to larger epicentral distance, while phases recorded at distances shorter than $120^{\circ}$ are reflected from a high-velocity "inner" core. Dis-

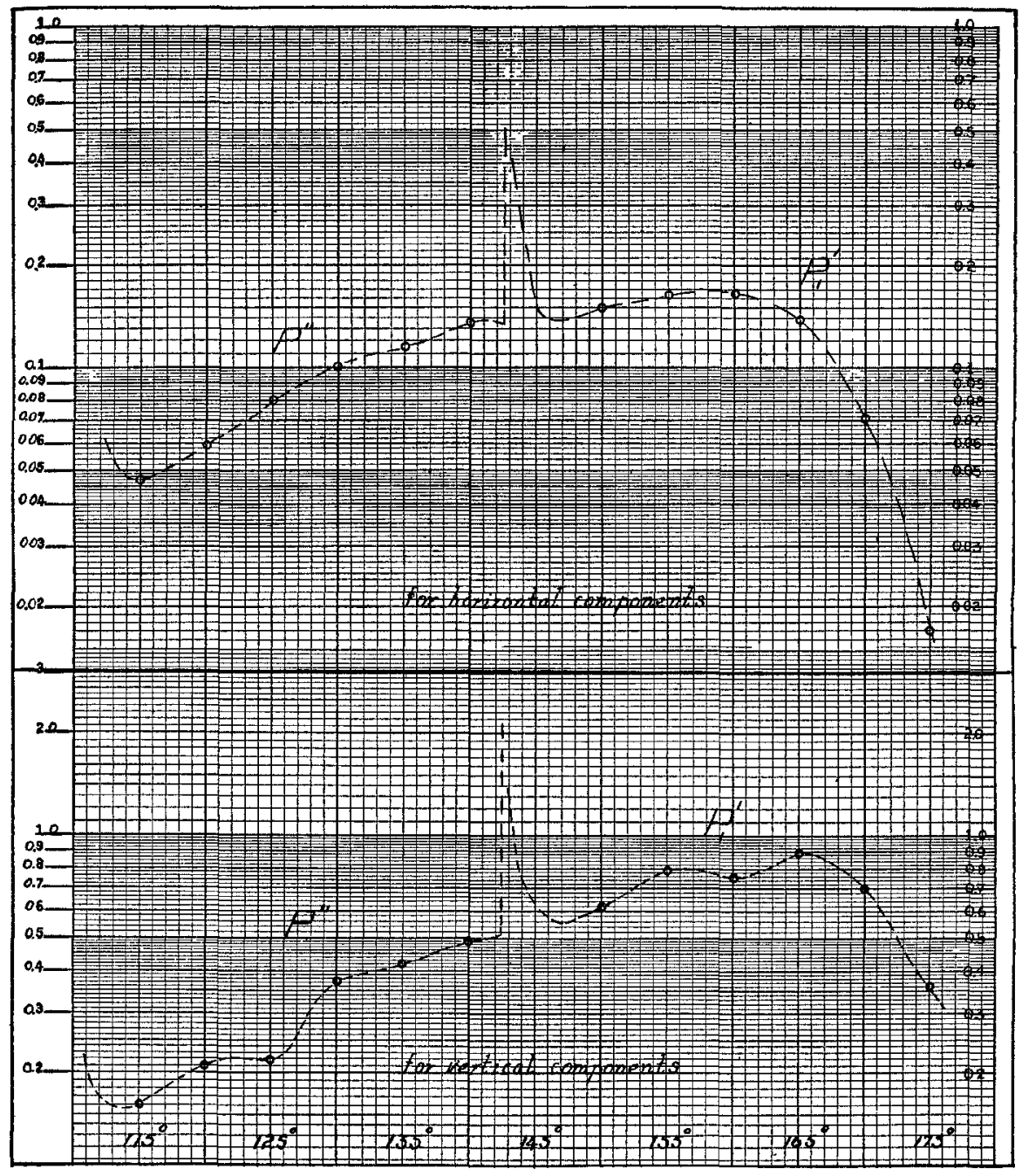

$$
\text { Fig. 7. } \frac{(u, w / T) P^{\prime \prime}, P_{1}{ }^{\prime}}{(u, w / T) P P} \text { vs } \Delta^{0} \text {. }
$$

continuities in period and energy relations at $120^{\circ}$ epicentral distance thus may reasonably be interpreted as a consequence of a velocity decrease followed by an abrupt increase at the "inner"-core transition zone as calculated by Jeffreys (1939b).

Possibly an increase in the velocity over the same finite interval of depth might also produce the observed results. In this case, shorter-period compo- 


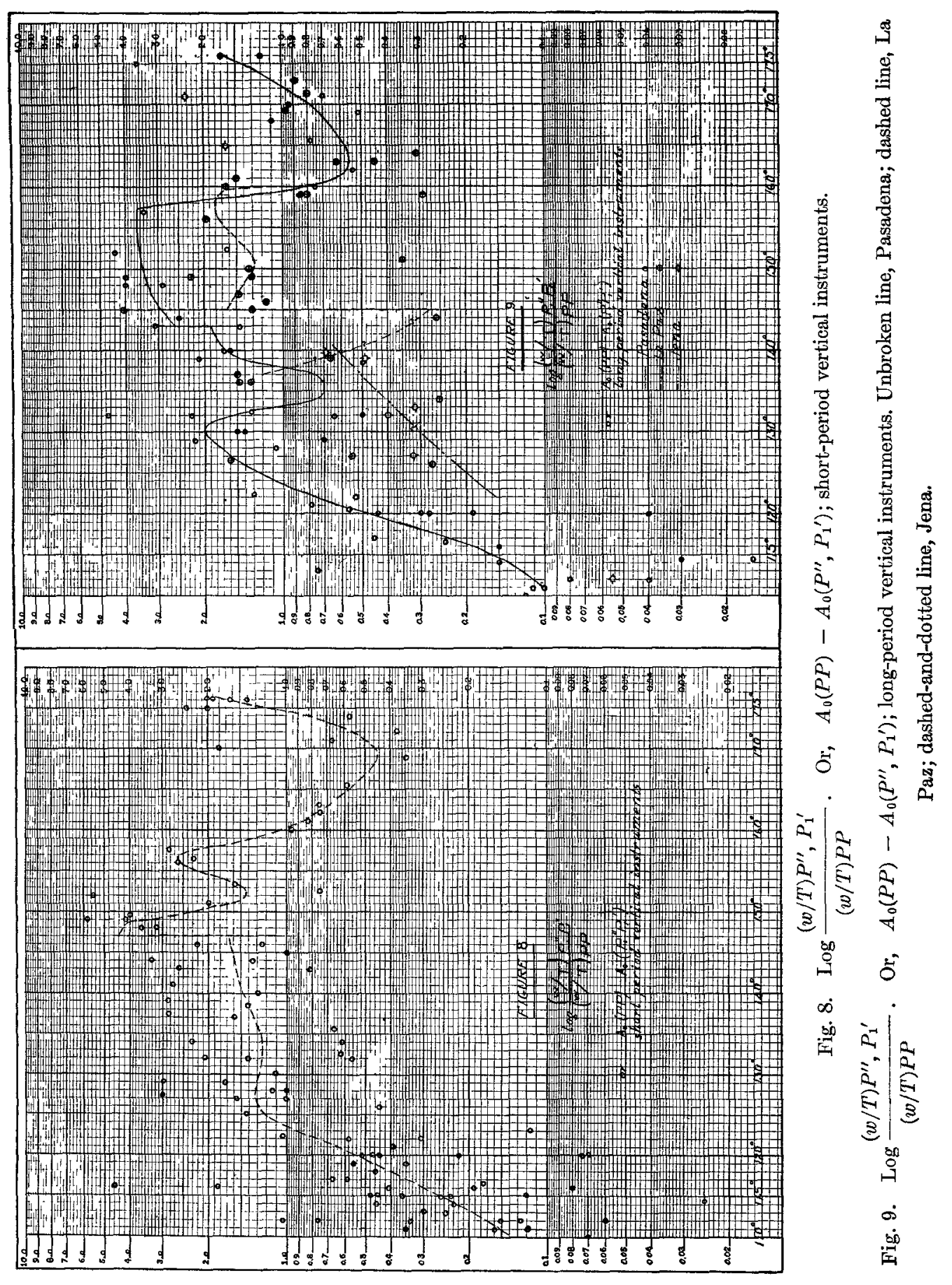


nents might be diffracted by the highly curved "inner" core so as to arrive on the surface at distances between $110^{\circ}$ and $120^{\circ}$. Longer-period components of motion, which have been noted at distances less than $110^{\circ}$ and with later arrival times (by reason of dispersion or length of path), may be diffracted or reflected waves.

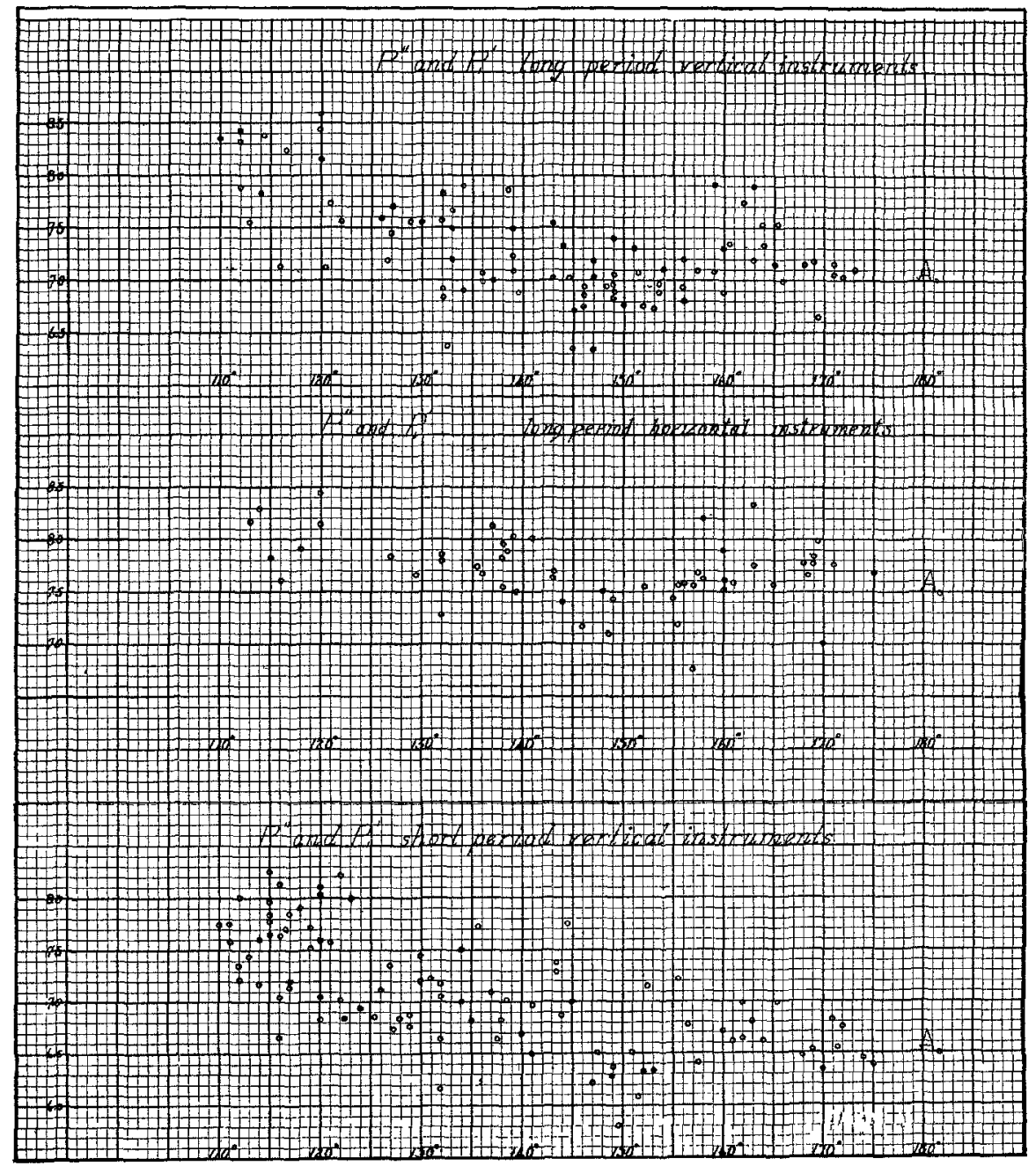

Fig. 10. $A_{0}$ vs $\Delta^{\circ} ; A_{0}=M-\log (u, w / T)-G(M-7)$.

An example of the variations in response which are found in the $145^{\circ}$ focal zone recordings are shown in figure 4 . Data show that the main focusing effect occurs near $145^{\circ}$ for waves recorded by long-period instruments. The extension to $143^{\circ}$ is based upon few observations. Displacement ratios of the latestarriving $\mathrm{P}^{\prime}$ group in the focal zone are higher than the second-arriving $\mathrm{P}^{\prime}$ focal zone motion by factors of about 3 . It is indeed possible that focal zone observations are affected by diffraction phenomena in accordance with Airy's 
theory. Jeffreys (1939a, p. 553) has shown that appreciable long-period amplitudes may persist up to about $14^{\circ}$ from the position of the caustic.

Quite clearly the $P_{1}{ }^{\prime}$ data of figures 8,9 , and 10 display a shadow zone of angular distances greater than $157^{\circ}$, one which is most prominent for longperiod motion. This drop in energy is believed to indicate that the point $\mathrm{C}$,

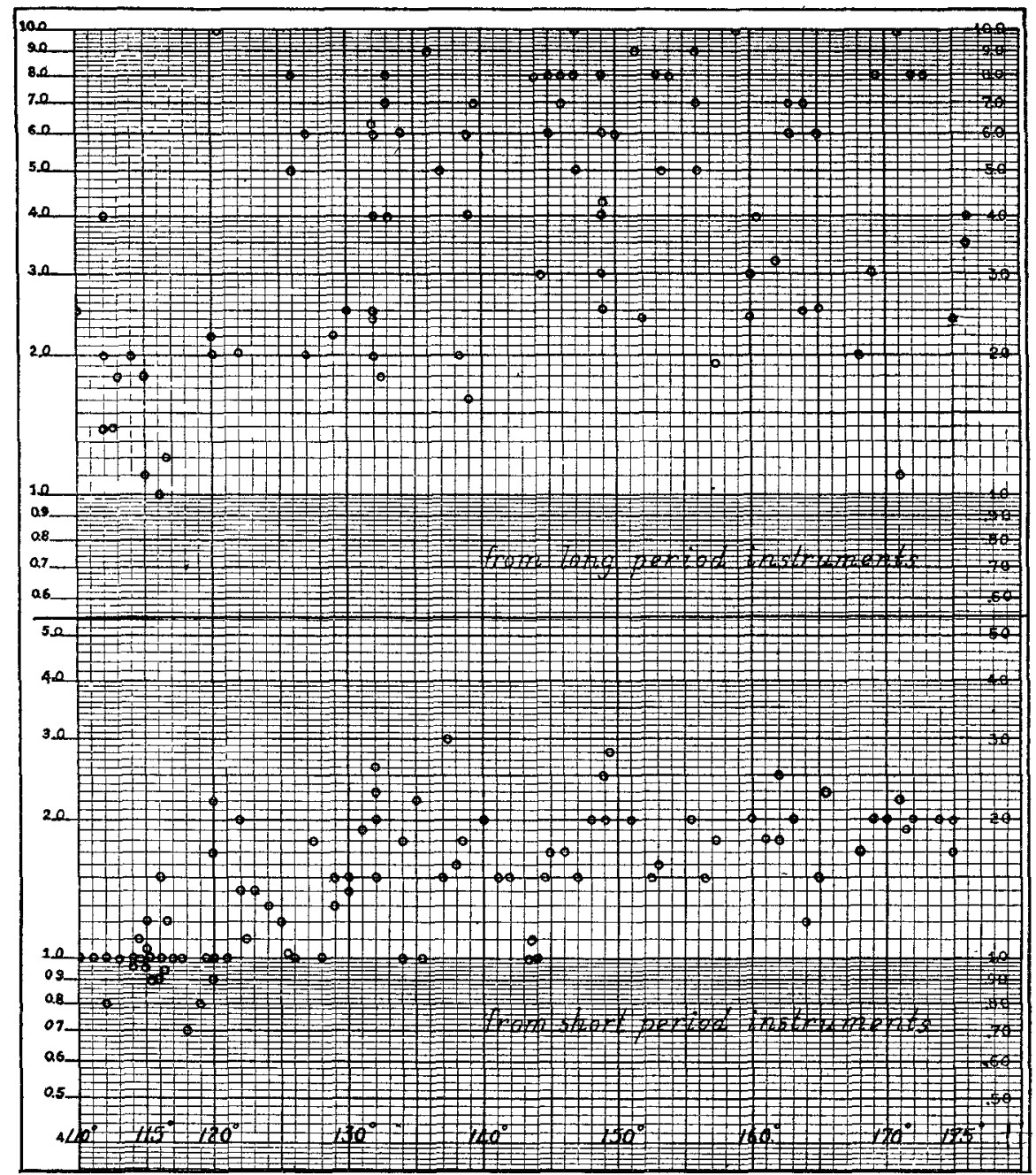

Fig. 11. T (sec.) vs $\Delta\left(^{\circ}\right)$ for $P^{\prime \prime}$ and $P_{1}^{\prime}$.

figure $1, a$, lies at or near $157^{\circ}$. In time this point must lie close to the $\mathrm{P}_{1}{ }^{\prime}$ curve. By the method of Wadati and Masuda (1934) the path relationships of those long-period waves which arrive at $157^{\circ}$ have been approximated. This analysis, while necessarily qualitative in light of the unsmoothed travel-time data used, indicates that those rays which arrive near $157^{\circ}$ graze the "inner" core. From similar travel-time considerations those short-period rays which emerge near $120^{\circ}$ appear to enter the core at about the same locality as the long-period 
rays which emerge near $157^{\circ}$. To determine points of entry into and exit from the core, it is assumed that the path of travel of each ray component of motion has a line of symmetry through the center of the earth.

Energy (here actually represented as the square root) contained in the shortperiod motion (fig. 10) at epicentral distances greater than $147^{\circ}$ diminishes with increasing distance. Over this same distance interval, a relative level of energy is maintained by the longer-period recorded motions. This variation suggests that the short- and long-period components of each ray in that portion of the wave front represented by $\mathrm{BC}$ in figure 1 do not travel the same path. Nor do the short- and long-period components of a ray appear to arrive at the same location on the surface of the earth. This is substantiated by the fact that the $120^{\circ}$ short-period and $157^{\circ}$ long-period energy-carrying rays appear to enter the core in approximately the same location. From this point of view it seems that, from the deeper penetrating rays in the $\mathrm{BC}$ portion of the wave, shorter-component periods would be refracted to shorter epicentral distances; from the shallower penetrating rays in the $\mathrm{BC}$ portion of the wave, components of motion longer in period than those refracted from the deeper penetrating rays would be refracted to epicentral distances which are greater than the epicentral distances reached by the shorter-period components. As discussed, the reversed segment $\mathrm{CD}$, figure 1, would exist for epicentral distances between $120^{\circ}$ and $157^{\circ}$.

Thus, the radiating energy contained in the narrow cone-shaped portion of the wave front called $\mathrm{BC}$ would reasonably be expected to distribute itself over a considerable range of epicentral distance. Surface distribution and the character of recorded motion might well be determined chiefly by four factors: 1 , the energy spectrum at the source; 2 , dispersion or the manner of grouping of the energy spectrum with time and distance; 3 , depth of penetration into the core; 4 , velocity-depth distribution within the core.

Characteristically the $\mathrm{P}^{\prime}$ motion is recorded as a series of short impulses. A typical example is shown in figure 6 . This recorded motion might be analyzed into a finite number of frequencies. Possibly this may suggest that, during propagation, component frequencies or groups which are governed by the mechanics of dispersive media are continuously drained or refracted from the main wave in accordance with velocity variations. Overlapping en échelon segments in the travel-time curve for $\mathrm{P}^{\prime \prime}$, the discontinuous increase of period with distance, and anomalous $\mathrm{P}^{\prime \prime}$ displacement ratios appear reasonable when viewed in this light.

The data which have been studied are believed to indicate that paths of travel for longer-period waves through the core are, with modifications, similar to those portrayed by Gutenberg $(1932$, p. 62). The form of the travel-time curve for long-period waves is believed similar to that postulated by Jeffreys and Bullen (Bullen, 1947, p. 175). However, transmission of long-period PKP energy through the "inner" core is possibly complicated by reason of diffraction or scattering of the incident wave by the curved "inner" core surface. Current data are also believed to indicate that for short-period motion the reversed segment (CD, fig. 1) does exist by reason of refraction. The refraction 
process whereby short-period waves arrive at shorter epicentral distances $\left(120^{\circ}\right.$ to $\left.147^{\circ}\right)$ and the paths of travel of these waves are different from those postulated by Gutenberg and Richter (1939). Current data are further believed to indicate that path relationships for short-period waves at epicentral distances between $110^{\circ}$ and $120^{\circ}$ are similar to those postulated by Jeffreys $(1939 b)$.

\section{SUMMARY}

Data taken in this study indicate that the epicentral distance of the main focal point of longitudinal waves through the earth's core varies with the period of recorded motion. For long-period waves this focal point appears to occur near $143^{\circ}$, while for short-period motion the focus extends to around $147^{\circ}$. The relations of energy-carrying period to epicentral distance and travel time may reasonably be attributed to dispersion. Whether dispersion may be normal or abnormal cannot yet be stated. Present results indicate that the longer-period wave groups travel more slowly or have a longer path of travel.

Fnergy relationships in addition to time-distance and period data at epicentral distances around $120^{\circ}$ to $125^{\circ}$ and at about $157^{\circ}$ suggest a discontinuity at the "inner" core transition zone. The discontinuity at $157^{\circ}$ is believed to correspond to the point of reversal in the travel-time curve for waves through the outer core (point C, fig. 1), and is believed to be determined by the size of the "inner" core. The discontinuity at $120^{\circ}$ is determined by the velocity-depth distribution at the "inner"-core contact and is believed to denote the end of the refracted, short-period segment, $\mathrm{CD}$ in figure 1 . The short-period waves arriving at epicentral distances between $120^{\circ}$ and $147^{\circ}$ and the long-period waves arriving between $145^{\circ}$ and $157^{\circ}$ appear to have been separated from each other during their passage through the earth. Presumably these separate waves leave the source as one wave or as one composite energy spectrum distribution. Their separation may be effected by dispersion in the mantle, by refraction on passing into the core, or by dispersion within the core. Arrival at different epicentral distances is a consequence of velocity distribution within the earth.

These data do not permit definition of the velocity variations which exist between the outer and the "inner" core. However, results appear reasonable when viewed in the light of a velocity decrease at the "inner" core transition zone followed by an abrupt increase. A velocity increase over this same transitional zone is not precluded by results of this investigation.

\section{ACKNOWLEDGMENTS}

The author wishes to acknowledge help received from Dr. B. Gutenberg and from Dr. C. F. Richter. Many others at the Seismological Laboratory contributed toward completion of this work. Financial aid provided by the Stanolind Oil and Gas Fellowship is gratefully acknowledged. 


\section{REFERENCES}

BULLEN, K. E.

1947. An Introduction to the Theory of Seismology, Cambridge University Press.

DANA, S. W.

1944. "The Partition of Energy among Seismic Waves Reflected and Refracted at the Earth's Core," Bull. Seism. Soc. Am., Vol. 34, no. 4, pp. 189-197.

GUTENBERG, B.

1914. "Ueber Erdbebenwellen," VIIA, Nachr. Ges. Wiss. Göttingen, math.-phys. Kl., p. 1.

1932. Handbuch der Geophysik, Bd. 4, "Erdbeben," Berlin, 1932.

1944. "Energy Ratio of Reflected and Refracted Seismic Waves," Bull. Seism. Soc. Am., Vol. 34, No. 2, pp. 85-102.

1945. "Amplitudes of P, PP, and S, and Magnitudes of Shallow Earthquakes," Bull. Seism. Soc. Am., Vol. 35, No. 2, pp. 57-69.

Gutenberk, B., and C. F. Richter

1938. "P' and the Earth's Core," Mon. Not. Roy. Astron. Soc., Vol. 4, No. 5, pp. 363-372.

1939. "On Seismic Waves" (fourth paper), Gerlands Beitr. Geophys., Vol. 54, pp. 94-136.

1949. Seismicity of the Earth, Princeton University Press.

JEFFREYS, $\mathrm{H}$.

1939a." "The Times of Core Waves," Mon. Not. Roy. Astron. Soc., Vol. 4, No. 7, pp. $548-561$.

1939b. "The Times of Core Waves" (second paper), Mon. Not. Roy. Astron. Soc., Vol. 4, No. 8, pp. 594-615.

KNotT, C. G.

1899. "Reflexion and Refraction of Elastic Waves, with Seismological Applications," Phil. Mag., 5th ser., pp. 64-97.

Lehmann, I.

1937. "P'," Publ. Bur. Cent. Séism. Internat., Série A: Travaux scientifiques, fase. 14, pp. 87-115.

Richter, C. H.

1935. "An Instrumental Earthquake Magnitude Scale," Bull. Seism. Soc. Am., Vol. 25, pp. 1-32.

WADATI, K. and MASUDA, K.

1934. "On the Travel Time of Earthquake Waves, Part VI," Geophysical Magazıne, Tokyo, Vol. 8, pp. 187-194.

Zoeppritz, K., L. Geiger, and B. Gutenderg

1912. "Ueber Erdbebenwellen. V," Nachr. Gesell. d. Wiss. Göttingen, math.-phys. Kl., pp. 121-206.

California Institute of Technology

Division of the Geological Sciences

Pasadena, Calmornia

Contribution No. 551. 